\title{
Mixed Kalman-Fuzzy Sliding Mode State Observer in Disturbance Rejection Control of a Vibrating Smart Structure
}

\author{
Atta Oveisi and Tamara Nestorović \\ Ruhr-Universität Bochum, Mechanik Adaptiver Systeme, Institut Computational Engineering, D-44801, Bochum, \\ Germany.
}

(Received 30 March 2017; accepted 13 July 2019)

In the controllers that are synthesized on a nominal model of a nonlinear plant, the parametric matched uncertainties and nonlinear/unmodelled dynamics of the high order nature can significantly affect the performance of the closedloop system. On this note, owing to the robust characteristic of the sliding mode observer against modelling perturbations, measurement noise, and unknown disturbances and due to the non-fragile behaviour of the Kalman filter against process noise, a mixed Kalman sliding mode state-observer is proposed and later enhanced by the addition of an intelligent fuzzy agent. In light of the proposed technique, the chattering phenomena and the conservative boundary neighboring layer of the high gain sliding mode observer are addressed. Then, a robust active disturbance rejection controller is developed by using the static feedback of the estimated states using a direct Lyapunov quadratic stability theorem. The reduced order plant for control design purposes is subjected to some simulated square-integrable disturbances and is assumed to have mismatched uncertainties in the system matrices. Finally, the robust performance of the closed-loop scheme with respect to the mentioned perturbation signals and modelling imperfections is tested by implementing the control system on a mechanical vibrating smart cantilever beam.

\section{INTRODUCTION}

The uncertainty of the perturbation of the plant model from its real states may lead to unexpected behaviour of the closedloop system. Thus, it is vital to take into account the effects of such perturbations in the structure elements during the modelling process. ${ }^{1-3}$ Such a view leads to robustness of the predicted response of the system as well as increasing the applicability and reliability of the designed controller. On the other hand, the model-based regulators were developed based on the simplified mathematical replicas of the real plant. These two modelling requirements constrain the control methodologies derived based on the norm-bounded representations of unmodeled dynamics, parametric uncertainties, and exogenous disturbance. Thus, a robust controller may be considered as the main candidate that justifies these inquiries. ${ }^{4,5}$

Kalman filtering is extensively employed in state estimation. Nevertheless, it is recognized as unable to handle large modelling uncertainties. ${ }^{6,7}$ The sliding mode observer (SMO) was proposed to address this issue both for the dynamical systems with linear and nonlinear behaviour under large disturbance signals. Based on the classical sliding mode (SM) technology, a discontinuous switching function was utilized to hold the trajectory of the observation residuals on a manifold. Hence, the observation residuals became independent of the modelling perturbations in estimation error space. A central concern of the SM technique was the sensitivity of the control system to the uncertainties in the reaching phase, during which the error trajectory travelled toward the sliding surface. ${ }^{8}$ In recent years, some investigations were performed in order to examine the effects of these parametric uncertainties in the robust performance of SMO. ${ }^{9}$ A standard design configuration for SMO was based on the equivalent control (EC) theory. Accordingly, for nonlinear dynamical systems subjected to bounded uncertainties, the output observation error together with its high order derivative were employed to construct a sliding manifold. ${ }^{10}$

A SM controller (SMC) and observer have one common drawback, namely, the chattering phenomenon that excites the high-order dynamics of the system, which were mostly neglected in the design procedure. The second issue in the actuating element was the high control activity, non-smooth actuation, and actuation saturation in the SMC. ${ }^{11}$ An accepted workaround for this problem was modifying the switching function of SM technique to a neighbouring boundary function such as saturation function which lead to a finite steady-state error. $^{12}$ The neighbouring layer depth was dependent on the switching gain. Notably, if the system was exposed to a large disturbance amplitude and modelling perturbations, the SM system required a thick boundary layer to account for the chattering problem. Such a behaviour prevented the error dynamics to slide toward the SM manifold, and, therefore, a broad boundary layer was mostly undesirable in control systems. In contrast, an intelligent element such as a fuzzy set based on linguistic variables may transfer the knowledge of the SM expert to the observer element instead of a passive boundary neighbouring function. ${ }^{13}$ In this paper, a Mamdani fuzzy mapping was used to create a smooth switching function that can: i) handle the parametric uncertain terms in state, input, and output matrices in state space representation of the plant; ii) treat the nonlinear norm-bounded globally Lipschitz unmodelled dynamics; and iii) mimic the behaviour of boundary layer neighbouring function without the mentioned drawbacks. For this purpose, a nonlinear multi-input-multi-output (MIMO) system 
was linearized based on the idea of Rajamani, and then, a fuzzy SMO (FSMO) was added to the conventional Kalman filter as a complementary term in the dynamics of state-estimator. ${ }^{14}$ In addition to the asymptotic stability of the estimation error dynamics, the exponential convergence, and the disturbance rejection in estimation error were addressed carefully to provide a robust state observation framework.

The active disturbance rejection control (ADRC) was extensively researched in the last decades. ${ }^{15,16}$ ADRC may be interpreted as a frequent alteration of the classical feedback control theory such as PID controller which was further elaborated on in Gao. ${ }^{15}$ In this paper, as the second contribution, an ADRC is proposed based on the feedback of the observed states (mixed Kalman-FSMO). Thereupon, an appropriate $H_{\infty}$ constraint was adjured on the transfer function from disturbance to the desired output for the class of systems with parametric uncertainties in all system matrices. Most of the mentioned ADRC schemes are limited to some abstract plant dynamics. In contrast, an adaptive fuzzy integral SMC (FISMC) was developed by Wai et al. to control the position of an electrical servo drive. ${ }^{17}$ An adaptive FSMO for a class of nonlinear MIMO systems with application in the modular and reconfigurable robot system was proposed in Gholami and Markazi. ${ }^{18}$ Mechanical vibrating systems based on the concept of smart structures were also categorized as the potential case studies for observer-based ADRC. ${ }^{19,20}$ In this paper, the control method is realized on a smart vibrating clamped-free beam to evaluate the disturbance rejection, state estimation, and chattering prevention performance.

In the rest of the paper, $\mathfrak{I}$ represents the identity matrix with appropriate dimension, $\|$.$\| shows the H_{\infty}$ norm for systems, superscript $T$ denotes the transpose of a matrix or a vector, and $\mathfrak{R}$ stands for the set of real numbers. Additionally, $\mathfrak{R}_{\geq 0}^{n}, \mathfrak{R}_{>0}^{n \times n}$, and ${ }^{+} \mathfrak{R}_{\geq 0}^{n \times n}$ represent some time dependent vectors, matrices, and positive definite matrices with dimension of $n \times 1, n \times n, n \times n$ for $t \geq 0$, respectively. Moreover, $\forall x \in \mathfrak{R}: \operatorname{sgn}(x)$ represents the well-known scalar sign function and $\forall \mathcal{X}_{i} \in \mathfrak{R}^{n_{i} \times n_{i}}, i=1 \ldots j: \operatorname{blkd}\left(\mathcal{X}_{i}\right)$ represents the block diagonal matrix of $j$ square sub-matrices each of which has the dimension of $n_{i} \times n_{i}$. TT states for transpose of all previous terms e.g. $A+\cdots+B+T T=A+\cdots+B+(A+\cdots+B)^{T}$ for all matrices with appropriate dimensions. Also, $\mathcal{E}($.$) repre-$ sents the expected value of a random variable.

\section{PROBLEM FORMULATION}

Consider the nonlinear dynamics of a MIMO plant:

$$
\begin{array}{r}
\dot{x}(t)=f(x(t), u(t))+g_{1}(x(t)) \mathrm{w}(t)+h_{1}(x(t)) v(t) \\
+F(x(t), u(t)) ; \\
y(t)=l(x(t), u(t))+g_{2}(x(t)) \mathrm{w}(t)+h_{2}(x(t)) v(t) ;
\end{array}
$$

where, $x(t) \in \mathfrak{R}_{\geq 0}^{n}, u(t) \in \mathfrak{R}_{\geq 0}^{q}$, w $(t) \in \mathfrak{R}_{\geq 0}^{s}, v(t) \in \mathfrak{R}_{\geq 0}^{r}$, and $y(t) \in \mathfrak{R}_{\geq 0}^{p}$ were the state, input, square-integrable disturbance, 2-norm bounded white Gaussian noise, and output vectors at time $t \geq 0$, respectively. For the sake of tractability of the problem, it was assumed that $g_{2}(x(t))=0$ and $h_{2}(x(t))=0$. In addition, in order to put the system in the class of nonlinear Lipschitz systems, it was assumed that $f(.,):. \mathfrak{R}_{\geq 0}^{n} \times \mathfrak{R}_{\geq 0}^{q} \rightarrow \mathfrak{R}_{\geq 0}^{n}, g_{1}():. \mathfrak{R}_{\geq 0}^{n} \rightarrow \mathfrak{R}_{\geq 0}^{s}$, $h_{1}():. \mathfrak{R}_{\geq 0}^{n} \rightarrow \mathfrak{R}_{\geq 0}^{r}$, and $l(.,):. \mathfrak{R}_{\geq 0}^{n} \times \mathfrak{R}_{\geq 0}^{q} \rightarrow \mathfrak{R}_{\geq 0}^{p}$ were sufficiently smooth and differentiable. Then, the necessary and sufficient conditions on the asymptotic stability of the observer-based control system could be addressed by the eigenvalues of the linearized matrices. ${ }^{14}$ Unstructured, unmodelled dynamics of the system were parameterized in $F(.,):. \mathfrak{R}_{\geq 0}^{n} \times \mathfrak{R}_{>0}^{q} \rightarrow \mathfrak{R}_{\geq 0}^{n}$ as a Lipschitz nonlinearity $\left(\|F(x, u)-F(\hat{x}, u)\|_{2} \leq f_{m}\|x-\hat{x}\|\right)$ with the positive Lipschitz constant $f_{m} \in{ }^{+} \mathfrak{R}$, in which $\hat{x}$ was the estimation of the states. For causal system $(1), f(0,0)=0$, with globally Lipschitz continuous dynamics, (1) could be linearized in the form of Eq. (2):

$$
\begin{array}{r}
\dot{x}=(A+\Delta A) x+(B+\Delta B) u+G \mathrm{w}+H v+F ; \\
y=(C+\Delta C) x ;
\end{array}
$$

where,

$$
\begin{gathered}
A=\frac{\partial f(x, u)}{\partial x} ; \quad B=\frac{\partial f(x, u)}{\partial u} ; \quad G=\frac{\partial g_{1}(x)}{\partial x} ; \\
H=\frac{\partial h_{1}(x)}{\partial x} ; \quad C=\frac{\partial l(x, u)}{\partial x} ;
\end{gathered}
$$

Additionally, $\Delta A \in \mathfrak{R}_{\geq 0}^{n \times n}, \Delta B \in \mathfrak{R}_{\geq 0}^{n \times q}$, and $\Delta C \in \mathfrak{R}_{\geq 0}^{p \times n}$ were the time-dependent perturbation terms that were associated with parametric uncertainty of modelling. In Eq. (2), and the rest of the paper, for simplifying the notation, the dependence of the system terms on time was suppressed. The perturbation terms were decomposed in form of $\Delta \mathfrak{G}=$ $M_{\mathfrak{G}} \Gamma_{\mathfrak{G}} N_{\mathfrak{G}} ; \forall \mathfrak{G}: A, B, C$, where $M_{\mathfrak{G}}$ and $N_{\mathfrak{G}}$ were known matrices with appropriate dimensions and $\Gamma_{\mathfrak{G}}: \mathfrak{R}_{\geq 0}^{n \times n}$ were the time-dependent unknown matrices that satisfied $\Gamma_{\mathfrak{G}}^{T} \Gamma_{\mathfrak{G}} \leq \mathfrak{I}$.

Remark 1. In Eq. (2), mathematically, $\Delta A$ could be converted to or included in the form of unmodelled dynamics $(F)$ and the main purpose of this decomposition was the available uncertainty quantification methods in the structural dynamical modelling. ${ }^{21,22}$

In this paper, the novel methods in uncertainty quantification were considered in the control development as one inspiration point to realize the gain variations in a plant in a practical manner. The interested reader may refer to Noël and Kerchen. ${ }^{23}$ Henceforth, the authors were active in developing a new mechanism for uncertainty quantification in mechanical structures with complex geometries that could be used as an alternative to conventional methods. ${ }^{21}$ Uncertainty quantification regarding the unmodelled dynamics of a high order nature was classically dealt with as lumped stable bounded time-varying functions. In terms of controller synthesis, such a view lead to conservative results in the controller design and closed-loop performance. However, analytical modelling of simpler geometries under large vibration amplitudes handed the structure uncertainty. Next, a combination of parametric identification methods and practical approaches that provide time-dependent responses of the nonlinear system made an efficient tool available for creating global models. The importance of considering such a parametric uncertainty depended on the DRC's application. In view of that matter, if the disturbance was active in the high-frequency range, then the high-order dynamics of the plant were more effective in the response of the system. ${ }^{24}$ 
In the application of vibration control, where the active methods were useful at frequencies up to $1 \mathrm{kHz}$, the introduction of unmodelled dynamics was crucial for addressing nonlinear damping models and nonlinear vibration. ${ }^{25}$ In contrast, the sensitivity of the controller/observer to the parametric uncertainties remained as the key constraint in robust control analysis due to the uncertainty propagation phenomenon. ${ }^{26}$ The bounded uncertainty of disturbance/noise input matrices $(G$ and $H$ ) were neglected in this paper since they may be considered as an additional source of disturbance. Additionally, the bound of the Euclidean norm of the disturbance was assumed to be $\overline{\mathrm{w}}$, i.e. there existed a positive real value that satisfied $\|\mathrm{w}\| \leq \overline{\mathrm{w}}$. By assuming the system to be stabilizable and detectable, the dynamics of the Kalman-SMO-based (KSMO) state-estimation mechanism were considered in the form of Eq. (4):

$$
\begin{array}{r}
\dot{\hat{x}}=A \hat{x}+B u+L_{K}(y-\hat{y})+L_{S M O} \operatorname{sgn}(y-\hat{y}) ; \\
\hat{y}=C \hat{x} ;
\end{array}
$$

in which, $\hat{x} \in \mathfrak{R}_{\geq 0}^{n}$ and $\hat{y} \in \mathfrak{R}_{\geq 0}^{p}$ were the estimated state- and output-vectors, correspondingly. The stability of the nominal observer was guaranteed by an unbiased Kalman filter algorithm with the Kalman gain $L_{K} \in \mathfrak{R}^{n \times q}$ based on the assumptions for $\mathrm{w}$ and $v$. The residual signal of the estimation was defined as $e=x-\hat{x}$. In Eq. (4), the sgn-term with SMO gain $\left(L_{S M O} \in \mathfrak{R}^{n \times q}\right.$ ) was assigned for compensating the effects of the disturbance, noise, and unknown bounded uncertainties of various nature. Hence, the hybrid KSMO system embraced the Kalman filter's term along with the discontinuous $\operatorname{sgn}($.$) ele-$ ment with separate design mechanisms. Using Eqs. (2-4), the dynamics of state-observation residuals could be derived as:

$$
\begin{aligned}
\dot{e}=(A & \left.-L_{K} C\right) e+\left(\Delta A-L_{K} \Delta C\right) x+\Delta B u \\
& +G \mathrm{w}+H v+F-L_{S M O} \operatorname{sgn}(y-\hat{y}) .
\end{aligned}
$$

Considering the control law for regulation problem as $u=$ $K \hat{x}$, in which $K \in \mathfrak{R}^{q \times n}$ was static observed-state feedback gain, the dynamics of the states-estimation error took the form of Eq. (6):

$$
\begin{array}{r}
\dot{e}=\left(A-L_{K} C-\Delta B K\right) e+\left(\Delta A-L_{K} \Delta C+\Delta B K\right) x \\
+G \mathrm{w}+H v+F-L_{S M O} \operatorname{sgn}(y-\hat{y}) .
\end{array}
$$

Next, the necessary and sufficient conditions for quadratic stability of the estimation system as well as the conditions on performance index were defined. As a result, the disturbance was rejected in the residual signal by introducing an appropriate objective on control system regarding control gain $K$ and SMO gain $L_{S M O}$

\section{MAIN RESULTS}

\subsection{Robust Stability and Performance of KFSMO}

The primary objective of this section is to formulate the quadratic stability of the state-estimation in Eq. (4) in terms of a convex feasibility/optimization problem. The outcome is the designed gain, $L_{S M O}$, which accounts for the parametric uncertainties, unmodelled dynamics, and disturbance. It is assumed that for the nominal system, $A-L_{K} C$ is a strictly Hurwitz matrix.

Lemma 1. The hybrid state estimator in Eq. (4) has a quadratic stable residual dynamics and satisfies the disturbance rejection on estimator regarding the $H_{\infty}$-norm bounded constraint on the transfer function $\left\|T_{e w}\right\|<\gamma_{w e}$, in which $\gamma_{w e} \in \mathfrak{R}$ is a strictly positive real value if there exist positive symmetric matrix $X \in \mathfrak{R}^{n \times n}$, matrices $\hat{K} \in \mathfrak{R}^{q \times n}$ and $L_{S M O} \in \mathfrak{R}^{n \times p}$, and $\epsilon_{i} \in+\mathfrak{R}, i=1 . .5$ such that simultaneously $H v<L_{S M O} \operatorname{sgn}(y-\hat{y})$ and the following matrix inequality is satisfied:

$$
\left[\begin{array}{ccc}
\Theta_{11} & \Theta_{12} & \Theta_{13} \\
\Theta_{12}^{T} & -\epsilon_{1} \mathfrak{I} & 0 \\
\Theta_{13}^{T} & 0 & \Theta_{33}
\end{array}\right] \leq 0
$$

where,

$$
\begin{array}{r}
\Theta_{11}=\left[\begin{array}{ccc}
S & \mathbf{0} & G \\
\mathbf{0} & \mathbf{0} & \mathbf{0} \\
G^{T} & \mathbf{0} & -\gamma_{w e}^{2} \mathfrak{I}
\end{array}\right] ; \quad \Theta_{12}=\left[\begin{array}{ccc}
\hat{K}^{T} N_{B}^{T} \\
\mathbf{0} \\
\mathbf{0}
\end{array}\right] ; \\
\Theta_{13}=\left[\begin{array}{cccc}
\mathbf{0} & \mathbf{0} & \mathbf{0} & \mathbf{0} \\
X N_{A}^{T} & X N_{C}^{T} & \hat{K}^{T} N_{B}^{T} & X \\
\mathbf{0} & \mathbf{0} & \mathbf{0} & \mathbf{0}
\end{array}\right] ; \\
\Theta_{33}=\operatorname{blkd}\left(-\epsilon_{2} \mathfrak{I},-\epsilon_{3} \mathfrak{I},-\epsilon_{4} \mathfrak{I},-\epsilon_{5} \mathfrak{I}\right) ; \\
+A^{T}+A X-X C^{T} L_{K}^{T}-L_{K} C X+\epsilon_{1} M_{B} M_{B}^{T} \\
M_{A} M_{A}^{T}+\epsilon_{3} L_{K} M_{C} M_{C}^{T} L_{K}^{T}+\epsilon_{4} M_{B} M_{B}^{T}+ \\
\epsilon_{5} f_{m}^{2} \mathfrak{I}+X .
\end{array}
$$

in which, $\mathbf{0}$ represents zero matrix with appropriate dimension. Then, the SMO gain $L_{S M O}$ and the controller gain $K=\hat{K} X^{-1}$ are calculated.

Proof. Consider the following Lyapunov function $\left(V_{e}(x, e)\right.$ : $\left.\mathfrak{R}_{\geq 0}^{n} \times \mathfrak{R}_{\geq 0}^{n} \rightarrow^{+} \mathfrak{R}_{\geq 0}\right)$ :

$$
V_{e}(x, e)=e^{T} P e+\int_{0}^{\infty}\left(e^{T} P e-\gamma_{w e}^{2} \mathrm{w}^{T} \mathrm{w}\right) d t .
$$

Then, by derivation of the Lyapunov function with respect to time, one can obtain:

$$
\begin{array}{r}
\dot{V}_{e}(x, e)=e^{T}\left(A^{T}-C^{T} L_{K}^{T}-K^{T} \Delta B^{T}\right) P e+x^{T}\left(\Delta A^{T}\right. \\
\left.-\Delta C^{T} L_{K}^{T}+K^{T} \Delta B^{T}\right) P e+\mathrm{w}^{T} G^{T} P e+v^{T} H^{T} P e \\
+F^{T} P e-\operatorname{sgn}(y-\hat{y})^{T} L_{S M O}^{T} P e+T T .
\end{array}
$$

Next, by using the assumptions on the structure of the perturbation matrices $\Delta A, \Delta B$, and $\Delta C$ and applying Lemma A.1, in Appendix A, on uncertain terms of Eq. (9) except for $F^{T} P e$, 
hands the following inequalities:

$$
\begin{gathered}
-e^{T} K^{T} \Delta B^{T} P e-e^{T} \Delta B K P e \leq \\
e^{T}\left(\epsilon_{1} K^{T} N_{B}^{T} N_{B} K+\epsilon_{1}^{-1} P M_{B} M_{B}^{T} P\right) e \\
x^{T} \Delta A^{T} P e+e^{T} P \Delta A x \leq \\
\epsilon_{2}^{-1} x^{T} N_{A}^{T} N_{A} x+\epsilon_{2} e^{T} P M_{A} M_{A}^{T} P e ; \\
-x^{T} \Delta C^{T} L_{K}^{T} P e-e P L_{K} \Delta C x \leq \\
\epsilon_{3}^{-1} x^{T} N_{C}^{T} N_{C} x+\epsilon_{3} e^{T} P L_{K} M_{C} M_{C}^{T} L_{K}^{T} P e ; \\
x^{T} K^{T} \Delta B^{T} P e+e^{T} P \Delta B K x \leq \\
\epsilon_{4} x^{T} K^{T} N_{B}^{T} N_{B} K x+\epsilon_{4}^{-1} e^{T} P M_{B} M_{B}^{T} P e ;
\end{gathered}
$$

Furthermore, by using the globally Lipschitz assumption on the unmodelled dynamics and employing Lemma A.2 in Appendix A, it is obvious that $F^{T} P e+e^{T} P F \leq x^{T}\left(\epsilon_{5}^{-1} \mathfrak{I}\right) x+$ $e^{T}\left(\epsilon_{5} f_{m}^{2} P^{2}\right) e$. By defining the augmented state vector as $x_{e}^{T}=\left[\begin{array}{lll}e^{T} & x^{T} & \mathrm{w}^{T}\end{array}\right]$ and using Eq. $(10), \dot{V}(x, e)$ can be transformed into:

$$
\begin{array}{r}
\dot{V}(x, e)=v^{T} H^{T} P e-\operatorname{sgn}(y-\hat{y})^{T} L_{S M O}^{T} P e \\
+T T+x_{e}^{T} \Theta x_{e} ;
\end{array}
$$

where,

$$
\Theta=\left[\begin{array}{ccc}
\theta_{11} & \mathbf{0} & P G \\
\mathbf{0} & \theta_{22} & \mathbf{0} \\
G^{T} P & \mathbf{0} & -\gamma_{w e}^{T} \mathfrak{I}
\end{array}\right]
$$

in which,

$$
\begin{array}{r}
\theta_{11}=A^{T} P+C^{T} L_{K}^{T} P+P A-P L_{K} C+\epsilon_{1} K^{T} N_{B}^{T} N_{B} K \\
+\epsilon_{1}^{-1} P M_{B} M_{B}^{T} P+\epsilon_{2} P M_{A} M_{A}^{T} P+\epsilon_{3} P L_{K} M_{C} M_{C}^{T} L_{K}^{T} P \\
+\epsilon_{4}^{-1} P M_{B} M_{B}^{T} P+\epsilon_{5} f_{m}^{2} P^{2}+\mathfrak{I} \\
\theta_{22}=\epsilon_{2}^{-1} N_{A}^{T} N_{A}+\epsilon_{3}^{-1} N_{C}^{T} N_{C}+\epsilon_{4} K^{T} N_{B}^{T} N_{B} K+\epsilon_{5}^{-1} \mathfrak{I}
\end{array}
$$

Finally, by applying the Congruence Transformation $\operatorname{blkd}\left(P^{-1}, P^{-1}, \mathfrak{I}\right)$ on Eq. (11b), defining $P^{-1}=X$ and $K X=\hat{K}$, and the successive use of the Schur complement Lemma, LMI condition Eq. (7) can be obtained and this completes the proof.

Consequently, by guaranteeing the LMI condition as well as the condition on noise channel which can be expressed as $\|H\|\|v\|<\left\|L_{S M O}\right\|$ in a more conservative form, it is proven that the estimation error converges to zero and the disturbance

\begin{tabular}{|c|c|c|c|c|c|c|c|c|}
\hline & $e$ & NB & NM & NS & $\mathrm{Z}$ & PS & PM & PB \\
\hline$\dot{e}$ & & & & & & & & \\
\hline NB & & NB & NB & NB & NB & NM & NS & $\mathrm{Z}$ \\
\hline NM & & NB & NB & NB & NM & NS & Z & PS \\
\hline NS & & NB & NB & NM & NS & Z & PS & PM \\
\hline Z & & NB & NM & NS & $\mathrm{Z}$ & PS & $\mathrm{PM}$ & PB \\
\hline PS & & NM & NS & Z & PS & PM & PB & $\mathrm{PB}$ \\
\hline PM & & NS & Z & PS & PM & PB & PB & PB \\
\hline PB & & Z & PS & PM & PB & PB & PB & PB \\
\hline
\end{tabular}
is rejected in the state estimation error dynamics. Since the latter condition is time-dependent and there exists no information on the noise signal, the dynamics of the estimation system is enhanced by introducing a Fuzzy system instead of the discontinuous $\operatorname{sgn}($.$) term. Such a change reduces the$ conservatism on the KSMO system. Accordingly, an intelligent Fuzzy framework is employed to address the well-known chattering problem of the observer by revising its dynamic as shown in Eq. (12):

$$
\begin{array}{r}
\dot{\hat{x}}=A \hat{x}+B u+L_{K}(y-\hat{y})+L_{F S M O} S_{\mathrm{w}} ; \\
\hat{y}=C \hat{x} ;
\end{array}
$$

where $L_{F S M O} \in \mathfrak{R}^{n \times q}$ is the observer gain associated with switching function $S_{\mathrm{w}}: \mathfrak{R}_{\geq 0}^{n} \times \mathfrak{R}_{\geq 0}^{n} \rightarrow \mathfrak{R}_{\geq 0}^{n}$. In Eq. (12), the
Table 1. Fuzzy rules.

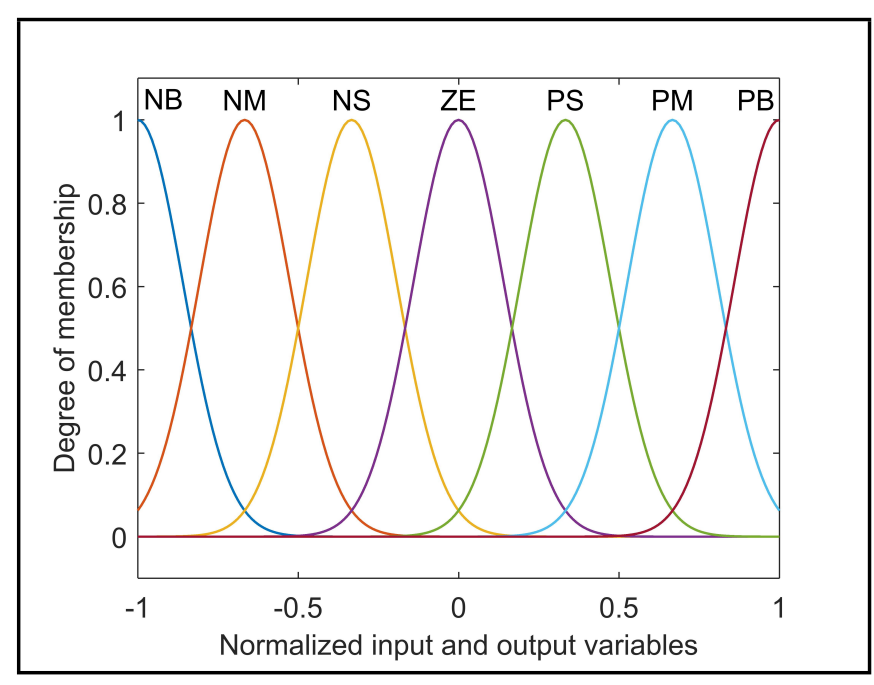

Figure 1. Membership functions of input/output fuzzy sets.

switching function $S_{\mathrm{w}}$ is constructed based on the system of if-then rules with two inputs of estimation error and its derivation w.r.t. time and the crisp output based on fuzzy logic of the expert. The generated rules are supposed to mimic the behaviour of $\operatorname{sgn}($.$) function. The fuzzy system realizes the non-$ linear mapping between the linguistic input variables and the crisp output as $S_{\mathrm{w}}=F S M O(e, \dot{e})$. In this paper, the input and output membership functions are assumed to be symmetric Gaussian. ${ }^{27}$ The fuzzy sets for inputs and outputs of $S_{\mathrm{w}}$ are labeled as P, Z, N, S, M, and B, which stand for Positive, Zero, Negative, Small, Medium, and Big, respectively. By normalizing both of the input linguistic variables in the range of $[-1,1]$, the membership function of input/output variables are presented in Fig. 1. Following the fuzzy rule table in Yau and Chen, Table 1 is created based on 49 if-then rules. ${ }^{28}$ The nonlinear mapping from the input linguistic variables to the output of $S_{\mathrm{w}}$ is presented in Fig. 2.

Corollary 1. The KFSMO of Eq. (12) has a quadratic stable state observation residual dynamics and rejects the disturbance on the state estimator in terms of the $H_{\infty}$-norm bounded constraint on transfer function $\left\|T_{e w}\right\|<\gamma_{w e}$, if there exist positive symmetric matrix $X \in \mathfrak{R}^{n \times n}$, matrices $\hat{K} \in \mathfrak{R}^{q \times n}$ and $L_{F S M O} \in \mathfrak{R}^{n \times p}$, and positive $\epsilon_{i} \in \mathfrak{R}, i=1 \ldots 5$ such that the following matrix inequality is satisfied:

$$
\left[\begin{array}{ccc}
\Phi_{11} & \Psi_{12} & \Phi_{13} \\
\Psi_{12}^{T} & \Psi_{22} & \mathbf{0} \\
\Phi_{13}^{T} & \mathbf{0} & \Phi_{33}
\end{array}\right] \leq 0 ;
$$




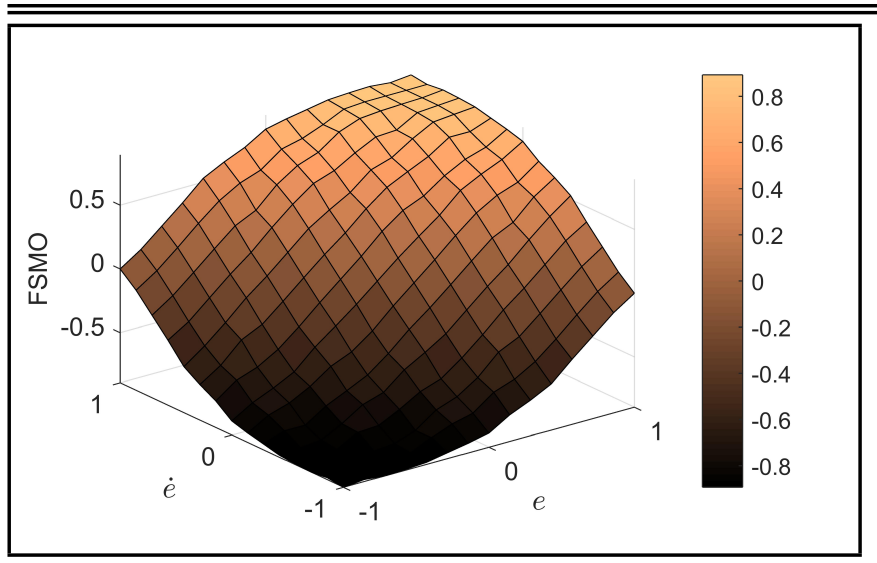

Figure 2. Fuzzy surface representing the nonlinear mapping between $e$ and $\dot{e}$ and $S_{\mathrm{w}}$.

where,

$$
\begin{array}{r}
\Psi_{12}=\left[\begin{array}{ccc}
H & -L_{F S M O} & \hat{K}^{T} N_{B}^{T} \\
\mathbf{0} & \mathbf{0} & \mathbf{0} \\
\mathbf{0} & \mathbf{0} & \mathbf{0}
\end{array}\right] \\
\Psi_{22}=\operatorname{blkd}\left(\mathbf{0}, \mathbf{0},-\epsilon_{1} \mathfrak{I}\right) .
\end{array}
$$

Then, the FSMO observer gain $L_{F S M O}$ and the controller gain $K=\hat{K} X^{-1}$ are calculated.

Proof. By using the same Lyapunov function as in Eq. (8) moreover, following the same steps as in Lemma 1, but, this time, defining the augmented vector as $x_{e}^{T}=$ $\left[\begin{array}{lllll}e^{T} & x^{T} & \mathrm{w}^{T} & v^{T} & S_{\mathrm{w}}^{T}\end{array}\right]$ and using the Congruence Transformation $\operatorname{blkd}(X, X, \mathfrak{I}, \mathfrak{I}, \mathfrak{I}, \mathfrak{I})$ and Schur complement Lemma, LMI (13a) can be obtained.

\subsection{ADRC based on KFSMO}

In this section, a second objective is introduced to address the ADRC on the desired output. Before moving to the main results, it should be noted that LMIs (7a) and (13a) are nonstrict ones which can be converted to strict feasible LMIs through eradicating the existing LME constraints and then reducing the resulted LMIs by eliminating any constant null space (see page 19 of Boyd et al.). ${ }^{29}$ Moreover, non-strict LMI constraints do not reduce the generality of the method since satisfying strict inequality constraints in applied optimization interfaces e.g. YAMIP are infeasible.

Bounded Real Lemma (BRL). ${ }^{30}$ For the following LTI system in the state-space form:

$$
\begin{aligned}
& \dot{x}=A x+B \mathrm{w} ; \\
& z=C x+D \mathrm{w} ;
\end{aligned}
$$

in which $z \in \mathfrak{R}_{\geq 0}^{m}$ is the objective function signal, for a prescribed strictly positive $\gamma \in{ }^{+} \mathfrak{R}$ and based on the performance index $J(z, \mathrm{w}): \mathfrak{R}_{\geq 0}^{n} \times \mathfrak{R}_{\geq 0}^{m} \rightarrow+\mathfrak{R}$ that is defined as $J(z, \mathrm{w})=\int_{0}^{\infty}\left(z^{T} z-\gamma^{2} \mathrm{w}^{T} \mathrm{w}\right) d t, J(z, \mathrm{w})<0$, if and only if there exist a symmetric positive definite matrix $\mathrm{E}$ known as Lyapunov function matrix such that the LMI condition (15) is satisfied:

$$
\left[\begin{array}{ccc}
A \mathrm{E}+\mathrm{E} A^{T} & \mathrm{E} C^{T} & B \\
C \mathrm{E} & -\mathfrak{I} & D \\
B^{T} & D & -\gamma^{2} \mathfrak{I}
\end{array}\right]<0 .
$$

Based on BRL, an equivalent Theorem 1 is developed to guarantee the asymptotic stability of the system based on the KFSMO (Eq. (13)):

Theorem 1. The uncertain system (2) with observer system (12) satisfies the $H_{\infty}$-norm bounded objective functions $\left\|T_{e w}\right\|<\gamma_{w e}$ and $\left\|T_{y w}\right\|<\gamma_{w c}$ if there exist symmetric positive definite matrices $X \in \mathfrak{R}^{n \times n}$ and $Y \in \mathfrak{R}^{n \times n}$, matrix $L_{\infty} \in \mathfrak{R}^{p \times n}$, positive constants $\epsilon_{i}, i=1 \ldots 9$, and $\hat{K} \in \mathfrak{R}^{q \times n}$ such that the following LMI/LME is satisfied:

$$
\begin{aligned}
& {\left[\begin{array}{ccc}
\Phi_{11} & \Psi_{12} & \Phi_{13} \\
\Psi_{12}^{T} & \Psi_{22} & \mathbf{0} \\
\Phi_{13}^{T} & \mathbf{0} & \Phi_{33}
\end{array}\right] \leq 0 ; \quad\left[\begin{array}{ccc}
\Pi_{11} & \Pi_{12} & \Pi_{13} \\
\Pi_{12}^{T} & \Pi_{22} & \mathbf{0} \\
\Pi_{13}^{T} & \mathbf{0} & -\epsilon_{9} \mathfrak{I}
\end{array}\right] \leq 0 ; } \\
& \\
&
\end{aligned}
$$

where,

$$
\begin{gathered}
\Pi_{11}=\left[\begin{array}{ccc}
\Sigma & -B \hat{K} & G \\
-\hat{K}^{T} B^{T} & \mathbf{0} & \mathbf{0} \\
G^{T} & \mathbf{0} & -\gamma_{\mathrm{wc}}^{2} \mathfrak{I}
\end{array}\right] ; \\
\Pi_{12}=\left[\begin{array}{cccc}
H & Y & Y N_{A}^{T} & \hat{K}^{T} N_{B}^{T} \\
\mathbf{0} & \mathbf{0} & \mathbf{0} & \mathbf{0} \\
\mathbf{0} & \mathbf{0} & \mathbf{0} & \mathbf{0}
\end{array}\right] ; \\
\Pi_{12}=\left[\begin{array}{c}
\mathbf{0} \\
\hat{K}^{T} N_{B}^{T} \\
\mathbf{0}
\end{array}\right] ; \\
\Pi_{22}=\operatorname{blkd}\left(\mathbf{0},-\epsilon_{6} \mathfrak{I},-\epsilon_{7} \mathfrak{I},-\epsilon_{8} \mathfrak{I}\right) ; \\
+A^{T}+A Y+B \hat{K}+\hat{K}^{T} B^{T}+\epsilon_{6} f_{m}^{2} \mathfrak{I}
\end{gathered}
$$

Then, the FSMO observer gain $L_{F S M O}$ and the controller gain $K=\hat{K} X^{-1}$ are calculated.

Proof. The equivalent form of BRL for Eq. (2) can be obtained by assuming the Lyapunov function as $V_{x}=x^{T} R x+$ $\int_{0}^{\infty}\left(z_{\infty}^{T} z_{\infty}-\gamma_{w c}^{2} \mathrm{w}^{T} \mathrm{w}\right) d t>0$ where $z_{\infty}=L_{\infty} x$ is the desired output. Then, by derivation of the Lyapunov function w.r.t. time and using Lemmas A.1 and A.2, the following inequalities can be stated:

$$
\begin{array}{r}
F^{T} R x+x^{T} R F \leq \\
x^{T}\left(\epsilon_{6}^{-1} \mathfrak{I}+\epsilon_{6} f_{m}^{2} R^{2}\right) x ; \\
x^{T} \Delta A^{T} R x+x^{T} R \Delta A x \leq \\
x^{T}\left(\epsilon_{7}^{-1} N_{A}^{T} N_{A}+\epsilon_{7} R M_{A} M_{A}^{T} R\right) x ; \\
x^{T} K^{T} \Delta B^{T} R x-x^{T} \Delta B K R x \leq \\
x^{T}\left(\epsilon_{8}^{-1} K^{T} N_{B}^{T} N_{B} K+\epsilon_{8} R M_{B} M_{B}^{T} R\right) x ; \\
e^{T} K^{T} \Delta B^{T} R x+x^{T} R \Delta B K e \leq \\
\epsilon_{9}^{-1} e^{T} K^{T} N_{B}^{T} N_{B} K e+\epsilon_{9} x^{T} R M_{B} M_{B}^{T} R x .
\end{array}
$$

Considering $\dot{V}_{e}=x_{c}^{T} \Pi x_{c}$ with $x_{c}^{T}=\left[\begin{array}{llll}x^{T} & e^{T} & \mathrm{w}^{T} & v^{T}\end{array}\right]$, and using the Congruence Transformation based on the block diagonal matrix blkd $\left(R^{-1}, R^{-1}, \mathfrak{I}, \mathfrak{I}\right), \Pi$ can be obtained as:

$$
\Pi=\left[\begin{array}{cccc}
Q_{11} & -B K Y & G & H \\
-Y K^{T} B^{T} & Q_{22} & \mathbf{0} & \mathbf{0} \\
G & \mathbf{0} & -\gamma_{w c}^{2} \mathfrak{I} & \mathbf{0} \\
H & \mathbf{0} & \mathbf{0} & \mathbf{0}
\end{array}\right]
$$


where,

$$
\begin{array}{r}
Q_{11}=Y A^{T}+A Y+Y K^{T} B^{T}+B K Y+\epsilon_{6}^{-1} Y^{2}+\epsilon_{6} f_{m}^{2} \Im \\
+e_{7}^{-1} Y N_{A}^{T} N_{A} Y+\epsilon_{7} M_{A} M_{A}^{T}+\epsilon_{8}^{-1} Y K^{T} N_{B}^{T} N_{B} K Y \\
+\epsilon_{8} M_{B} M_{B}^{T}+\epsilon_{9} M_{B} M_{B}^{T}+L_{\infty}^{T} L_{\infty} ; \\
Q_{22}=\epsilon_{9}^{-1} Y K^{T} N_{B}^{T} N_{B} K Y .
\end{array}
$$

Finally, by defining $K Y=\hat{K}$ and repetitive employment of Schur complement Lemma on all of the terms with $\epsilon_{6}^{-1}, \epsilon_{7}^{-1}$, $\epsilon_{8}^{-1}$, and $\epsilon_{9}^{-1}$ multipliers second LMI of Eq. (16) is obtained. The first LMI is the direct use of the results from Corollary 1. It should be mentioned that for output disturbance rejection an additional LME should be defined as $C Y-L_{\infty}=\mathbf{0}$. This completes the proof.

Remark 2. LME $X-Y=\mathbf{0}$ is the coupling term between the feasibility problem of the robust state estimation and robust ADRC.

Remark 3. By defining $\gamma_{w c}, \gamma_{w e} \in{ }^{+} \mathfrak{R}$ and replacing the associated terms $-\gamma_{w c}^{2} I$ and $-\gamma_{w e}^{2} I$ in LMI Eq. (16a) by $-\gamma_{w c} I$ and $-\gamma_{w e} I$, respectively, the maximum disturbance attenuation rate can be achieved by solving the following convex optimization problem:

Corollary 2. For the uncertain system (2) with observer system (12), the maximum disturbance attenuation based on the $H_{\infty}$-norm bounded objective functions $\left\|T_{e w}\right\|<\gamma_{w e}^{1 / 2}$ and $\left\|T_{y w}\right\|<\gamma_{w c}^{1 / 2}$ can be achieved if there exist symmetric positive definite matrices $X \in \mathfrak{R}^{n \times n}$ and $Y \in \mathfrak{R}^{n \times n}$, matrix $L_{\infty} \in \mathfrak{R}^{p \times n}$, positive constants $\epsilon_{i}, i=1 \ldots 9$, and $\hat{K} \in \mathfrak{R}^{q \times n}$ such that the following convex optimization problem has a solution:

$$
\min \alpha \gamma_{w c}+\beta \gamma_{w e}
$$

subject to LMI/LME system (16a) and (16b), in which, $\alpha$ and $\beta \in+\mathfrak{R}$ are user-defined values expressing the weight of the ADRC performance objective compared to robust KFSMO objective.

Remark 4. If an exponential convergence with the desired rate such as $\sigma_{c}, \sigma_{e} \in{ }^{+} \mathfrak{R}$ is prescribed by control engineer, then two additional constraints on the derivation of Lyapunov functions w.r.t. time should be added. Accordingly, in Corollary 1 and Theorem 1 the inequalities $\dot{V}_{k}() \leq.-2 \sigma_{k} V_{k}(), k:. e, c .^{31}$

\section{NUMERICAL EXAMPLE}

\subsection{Modelling of the Open Loop Plant}

In this section, by aiming at evaluating the performance of the proposed observer-based control system, the ADRC scheme was implemented on a mechanical vibrating plant. The objective of ADRC system was to suppress the oscillation of the beam at the plant output. The structure of the system, as presented in Fig. 3, consisted of a clamped-free aluminium beam with a Young's modulus $70 \mathrm{Gpa}$ and density $2.7 \mathrm{~g} / \mathrm{cm}^{3}$ and two piezo-patches bounded on one side of the structure. The controller and observer systems were calculated based on the results in Theorem 1. The closed-loop system configuration is presented in Fig. 4. The piezo-laminated beam plant has three inputs: the control inputs which acted on the twoactuator piezo-patches and the exogenous signal which excited the structure through an independent disturbance channel.

It should be mentioned that the torsional modes were neglected in the modelling of the beam since only the bending vibration suppression is analysed in this paper. The dynamics of the actuation was obtained by means of finite element analysis (FEA) in coupled electro-mechanical domain. Although it was assumed that the displacements were too small to satisfy the linear piezo-elasticity, in the case of large deformation (geometrical nonlinearities), the unmodelled nonlinear dynamics of the system could be addressed in the form of $F$ in Eq. (2). ${ }^{32}$ By assuming $\mathcal{M}, \mathcal{C}$, and $\mathcal{K}$ as the mass, damping, and stiffness matrices in the framework of FEM, the governing ordinary differential equation (ODE) of motion in matrix form could be written as $\mathcal{M} \ddot{q}+\mathcal{C} \dot{q}+\mathcal{K} q=\mathcal{F}$ with $q$ and $\mathcal{F}$ being the nodal states of displacement and electric potential and applied external inputs, respectively. The structure of the control effort was assumed to be $\mathcal{F}=\mathcal{B} u(t)$, where, $\mathcal{B}$ depended on the position of generalized control effort in FE structure. The output of the plant based on the nodal displacements and velocities was expressed as $y=C_{0 q} q+C_{0 v} \dot{q}$ with $C_{0 q}$ and $C_{0 v}$ being the modal output displacement and velocity matrices, respectively. The natural frequencies $\left(w_{i}\right)$ of the coupled structure as well as the mode shapes $\left(\phi_{i}\right)$ for $i=1 \ldots n$ was obtained by employing the classical harmonic solution $\left(q=\phi e^{\mathrm{i} w t}\right)$ and solving the obtained algebraic equation. Then, by applying the transformation $q=\phi q_{m}$, the nodal model of the plant could be transformed to the generalized modal displacement model, in which, $\phi$ was the matrix of eigen vectors. Finally, by using the proportional damping model, $\mathcal{C}=a \mathcal{M}+b \mathcal{K}$, orthogonality of mode shapes, and assuming the state vector as $x=\left[\begin{array}{ll}\mathbf{w} q_{m} & \dot{q}_{m}\end{array}\right]^{T}$, with $\mathbf{w}=\operatorname{diag}\left(w_{i}\right)$, the governing equation of motion could be presented in the state space form of Eq. (2). The interpretation of the state, input, and output matrices based on the FEA could be presented as Eq. (20):

$$
\begin{array}{r}
A=\left[\begin{array}{cc}
\mathbf{0} & \mathbf{w} \\
-\mathbf{w} & 2 \mathcal{Z} \mathbf{w}
\end{array}\right] ; \quad B=\left[\begin{array}{cc}
\mathbf{0} & B_{m}^{T}
\end{array}\right]^{T} ; \\
C=\left[\begin{array}{ll}
C_{m q} & C_{m v}
\end{array}\right] ;
\end{array}
$$

where $\mathcal{Z}=\operatorname{diag}\left(\zeta_{i}\right)$ with $\zeta_{i}$ being the damping ratio of $i$ th mode shape and $B_{m}=\phi^{T} B, C_{m q}=C_{0 q} \phi$, and $C_{m v}=$ $C_{0 v} \phi$.

Remark 5. In the state space representation of Eq. (20), the parametric uncertainty terms of Eq. (2) were not available, and their quantification was highly dependent on the operating frequency range of disturbance which acted on the structure. If the interference was active at high oscillation rates, then the effect of unmodelled/nonlinear dynamics in the nominal plant model was more important. In this frequency range, after efficient modelling of dynamics of low-frequency nature, the bound of the unknown additive uncertainty function in plant model could be obtained following the experimental method in Mignolet and Soize, and Adhikari et al. ${ }^{24,26}$ In contrast, if the frequency of the disturbance was not higher than the highest natural frequency of the modelled reduced order system, 


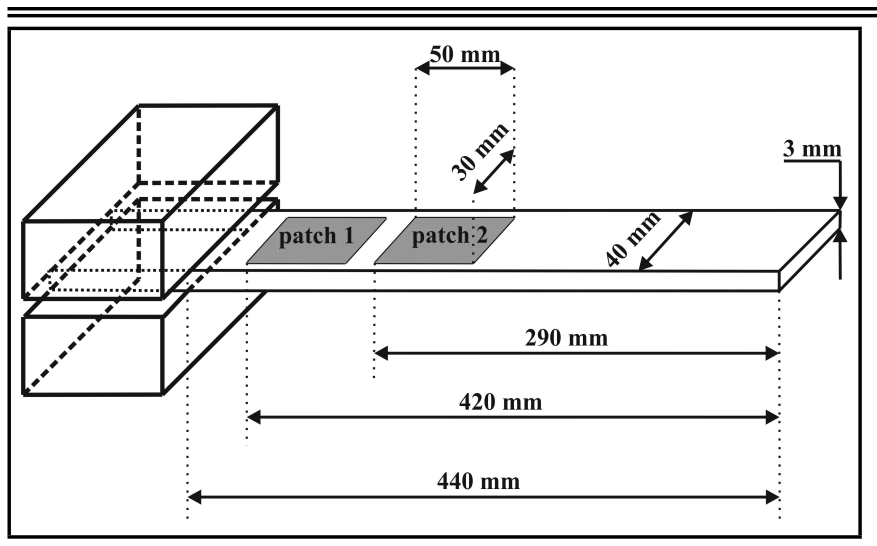

Figure 3. Geometry of the smart beam.

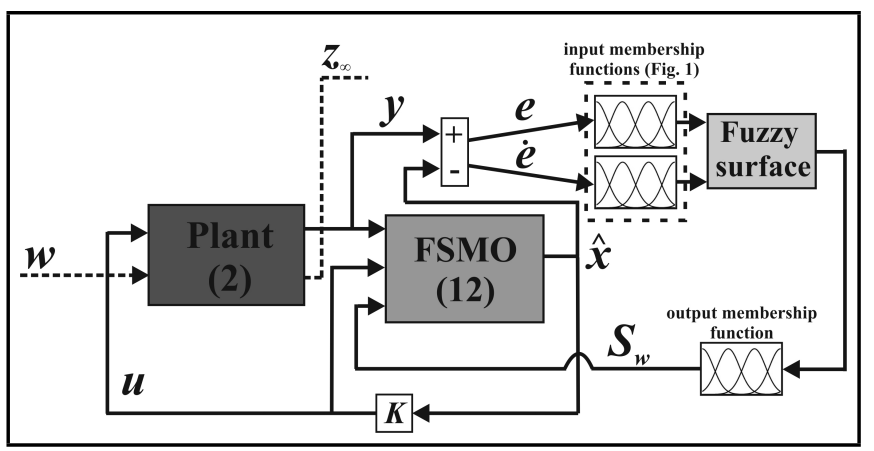

Figure 4. Schematic interconnection of the plant and KFSMO-based controller.

stochastic finite element method (SFEM) was an appropriate method for calculating the known matrices $\left(M_{A}, N_{A}, M_{B}\right.$ and $N_{B}$ ) in the mismatch uncertainty of $\Delta A$ and $\Delta B$, respectively. ${ }^{33}$

\subsection{Controller and Observer Design}

In this section, the numerical results of the employment of the proposed controller are presented on the piezo-laminated beam shown in Fig. 3. For design purposes, a reduced order system was obtained by considering three bending mode shapes of the smart structure. The three fundamental natural frequencies of the system were 96.7, 467.84, and 542.95 $\mathrm{rad} / \mathrm{sec}$ and their associated damping ratios were calculated as

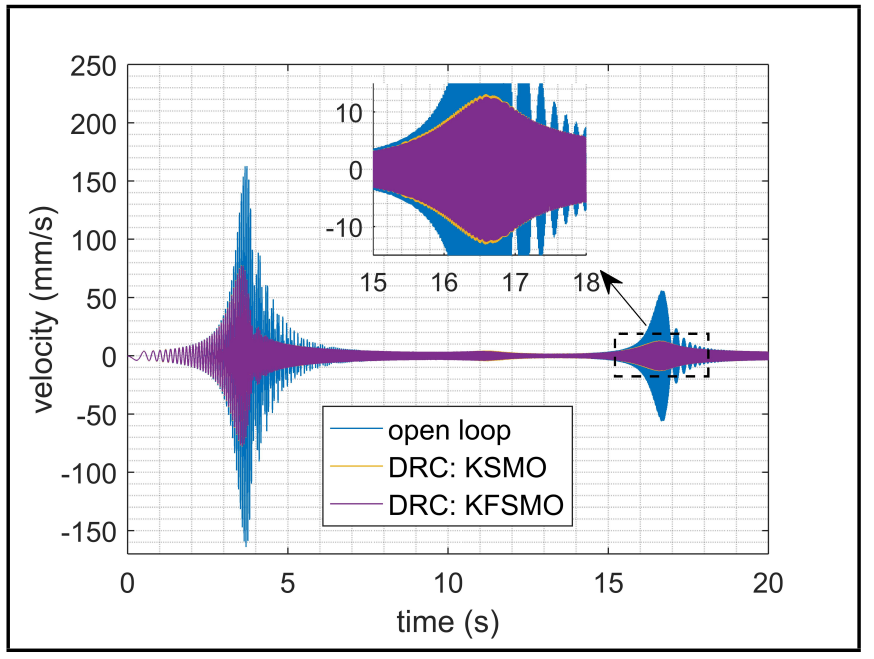

Figure 5. Comparison of measured outputs in time domain.
$1.25 \times 10^{-2}, 5.02 \times 10^{-3}$, and $3.79 \times 10^{-1}$, respectively. The remaining higher order modes were reflected in the form of the unstructured uncertainty of a norm-bounded time-dependent term. The nominal system was transformed in state space representation with the system matrices shown in Eq. (21), in which a maximum ten percent parametric variations were assumed in the form of, $\Delta A, \Delta B$, and $\Delta C$.

$$
\begin{gathered}
{\left[\begin{array}{ccccccc}
-1.29 & 106.68 & 0 & 0 & 0 & 0 \\
-106.48 & -1.8 & 0 & 0 & 0 & 0 \\
-0.39 & 0 & -2.58 & 514.6 & 0 & 0.03 \\
0.4 & -0.01 & -514.64 & -2.58 & 0 & 0 \\
0.14 & -0.13 & 0 & 0.01 & -226.23 & 552.53 \\
0.42 & 0 & 0 & 0 & -552.74 & -226.59
\end{array}\right] ;} \\
\\
{\left[\begin{array}{cccccccc}
-0.25 & 0.07 & -27.6 & 4.42 & -5.39 & -5.99 \\
-0.17 & 0.07 & 14.89 & -3.54 & 22.63 & 6.69
\end{array}\right]^{T} ;} \\
{\left[\begin{array}{lllllll}
-25.47 & 10.52 & 0.48 & -0.51 & -0.63 & -0.35
\end{array}\right] ;}
\end{gathered}
$$

with

$$
\begin{gathered}
{\left[\begin{array}{cccccc}
-0.02 & -1.35 & 0.07 & 2.8 & 3.71 & -3.86 \\
4.31 & 2.64 & 0.1 & -2.59 & -2.46 & 1.97 \\
-2.54 & 1.43 & 0.92 & -0.26 & 5.76 & 2.43 \\
1.23 & -4.18 & 4.7 & 2.39 & 2.45 & 2.17 \\
6.45 & -2.01 & -7.67 & 1.13 & 0.89 & 1.39 \\
-1.14 & -2.48 & 0.48 & -1.65 & 7.99 & -8.54
\end{array}\right] ;} \\
{\left[\begin{array}{ccccccc}
-1.65 & 0.92 & -2.2 & -1.08 & -0.77 & 1.4 \\
-1.75 & 2.12 & 5.17 & 4.09 & 5.1 & -1.34 \\
-0.95 & 0.5 & -3.62 & -0.67 & 1.69 & -4.16 \\
-0.11 & 2.75 & 0.5 & 0.25 & 6.96 & 2.15 \\
-0.35 & 0.32 & -0.76 & 5.18 & -2.1 & 0.64 \\
0.32 & -0.94 & -2.22 & 3.71 & 1.29 & 2.56
\end{array}\right] ;} \\
\left.\begin{array}{cccccccc}
0.06 & -0.2 & 0.11 & 0.05 & -0.3 & -0.17 \\
0.02 & -0.02 & -0.21 & -0.1 & 0.22 & 0.14
\end{array}\right]^{T} ;
\end{gathered}
$$

For examination of the robust enactment of the closed-loop system which included the parametric uncertainty of Eqs. (21a) and (21b) and $\|F(x, u)-F(\hat{x}, u)\|_{2} \leq 0.3\|x-\hat{x}\|$, an observer-based controller was designed. For this purpose, first, for the nominal deterministic model, the well-known Kalman filter was designed by solving the algebraic Riccati equation (ARE) of $L_{K}=\left(\mathfrak{P} C^{T}+\overline{\mathfrak{R}}\right) \mathcal{R}^{-1}$. In this equation, $\overline{\mathfrak{R}}=G \mathfrak{R}$ with $\mathfrak{R}=\mathcal{E}\left(\mathrm{w} v^{T}\right), \mathcal{R}=\mathcal{E}\left(v v^{T}\right)$, and $\mathfrak{P}$ was the steady-state error covariance $\left(\lim _{t \rightarrow \infty} \mathcal{E}\left(\{x-\hat{x}\}\{x-\hat{x}\}^{T}\right)\right)$. Accordingly, the obtained $L_{K}$ is presented in Eq. (22): 


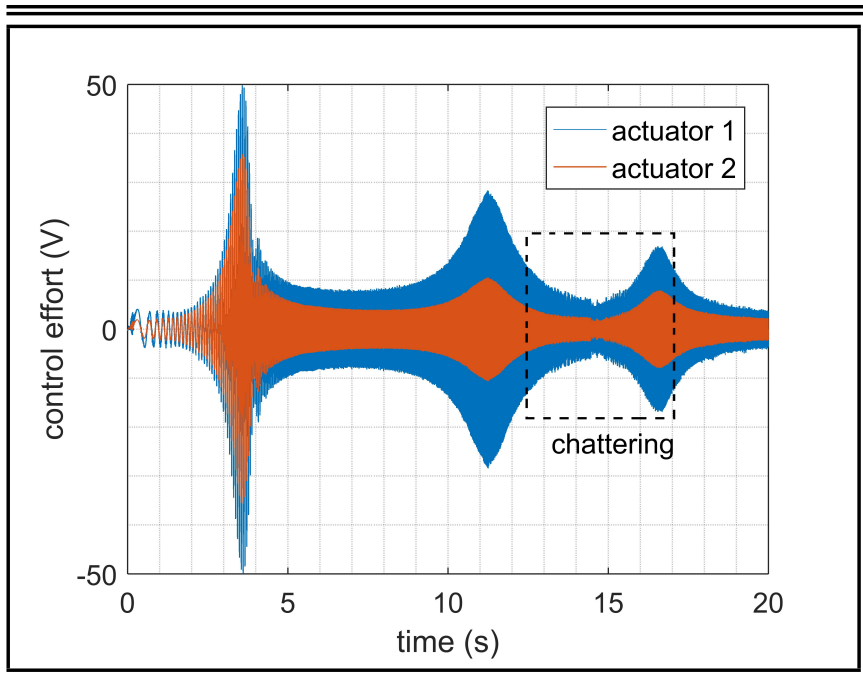

Figure 6. Control signal based on Kalman SMO.

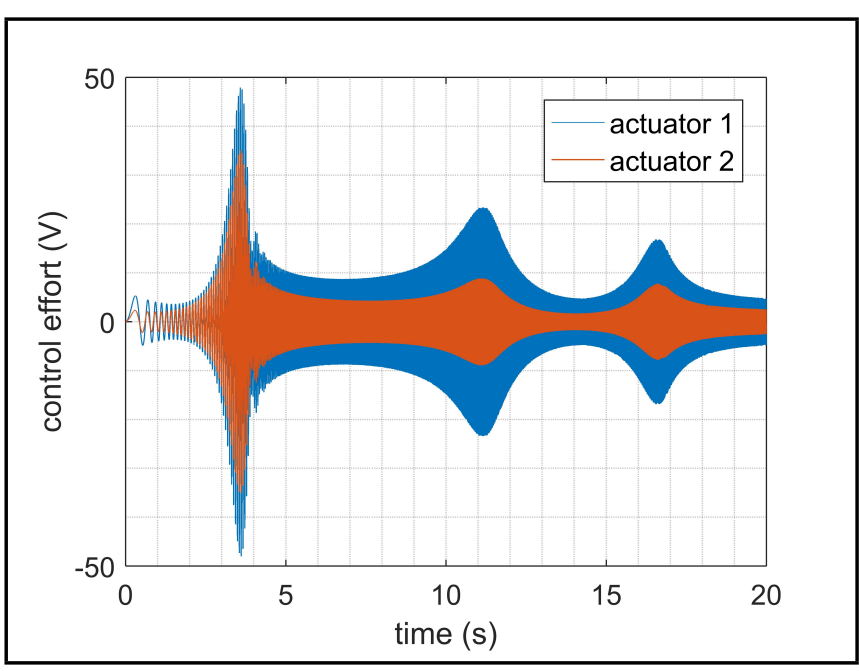

Figure 7. Control signal based on Kalman-Fuzzy SMO.

$L_{K}=$

$\left[\begin{array}{llllll}-59.48 & 31.75 & 3951.5 & -4057.8 & 6373.1 & -2161.9\end{array}\right]^{T}$.

Then, using the results from Theorem 1 (Eq. (16)), the controller gain and FSMO gain were obtained as:

$$
\begin{gathered}
{\left[\begin{array}{ccccccc}
16.05 & -2.14 & 1.33 & -0.42 & 0.61 & 0.33 \\
12.56 & -1.9 & 0.42 & 0.24 & 0.58 & 0.05
\end{array}\right]} \\
\\
{\left[\begin{array}{llllll}
0.08 & -0.02 & 0.53 & -0.66 & 1.11 & -1.18
\end{array}\right]^{T}}
\end{gathered}
$$

By implementing the control system based on the calculated gain $K$ in Eq. (23) and two observer gains $L_{K}$ and $L_{F S M O}$ on the plant with system matrices as in Eqs. (21), the prospect of the operational vibration attenuation performance was assessed. The optimal solution for LMI/LME system of Eq. (16) was acquired by using Scilab. Next, the disturbance channel was activated by realization of a chirp signal. The frequency of the disturbance signal was swept from zero to $100 \mathrm{~Hz}$ within a

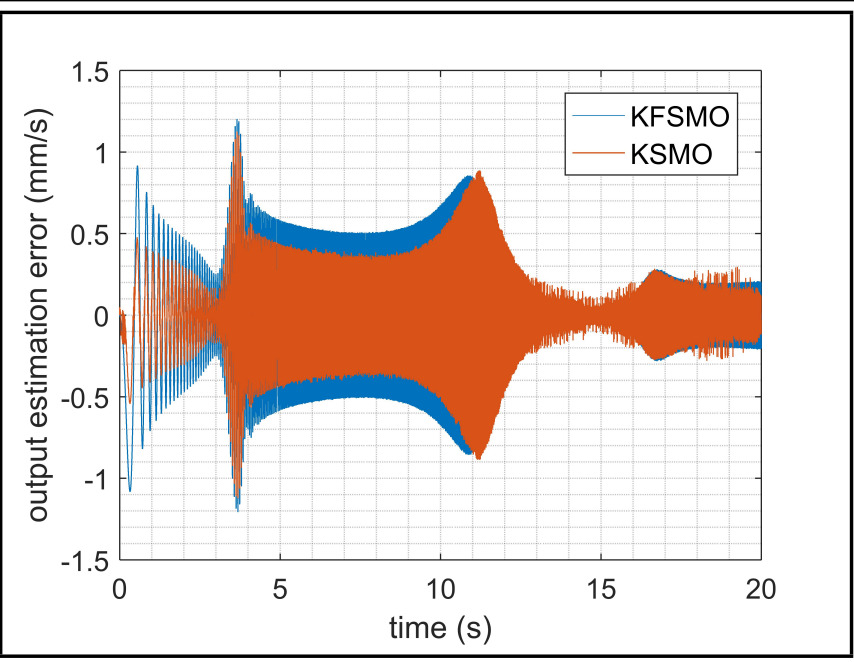

Figure 8. Estimation error of the closed-loop system: comparison between KSMO and KFSMO.

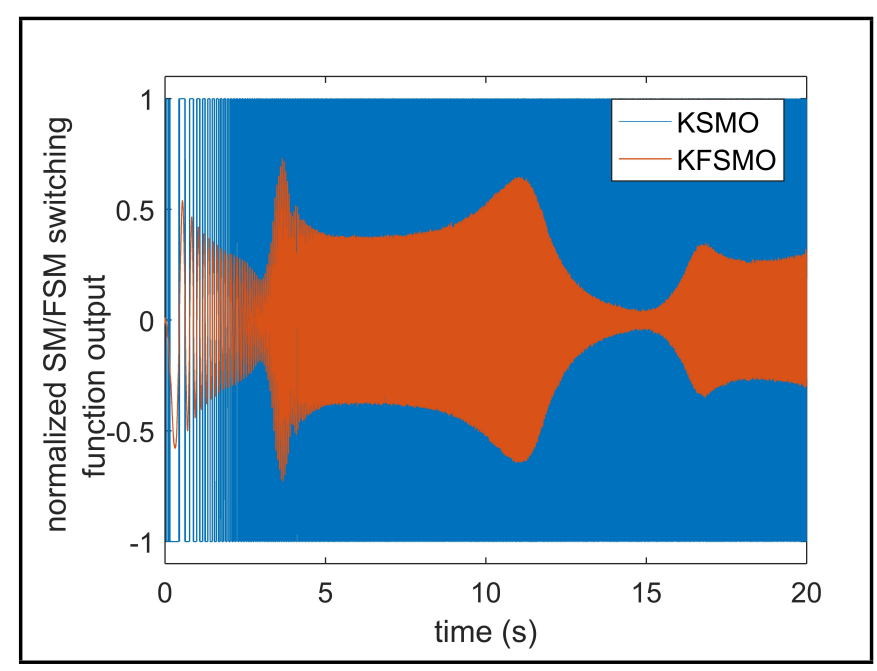

Figure 9. Switching functions in forced vibration control.

twenty-second-time window in two cases of the controlled and uncontrolled systems. Investigations were carried out on the structure in Fig. 3 in time-domain with the sampling frequency of $1 \mathrm{kHz}$. Both of the robust controller and KFSMO systems were modelled on SIMULINK platform with standard fixed step explicit ODE5 solver (Dormand-Prince method). The response of the system for the close-loop and open loop cases is shown in Fig. 5 in time-domain based on the velocity measurement signal.

It could be seen that the control system based on the uncertain plant, suppressed the vibration magnitude within the considered frequency range. However, the quality of the ADRC using KFSMO (Eq. (12)) was better than the one based on KSMO by a small margin. Additionally, the applied control signals that are implemented through piezo-modules are depicted in Figs. 6-7.

Figure 6 shows that the control efforts that are actuated by each of the piezo-transducer based on KSMO have a chattering behaviour in the region indicated inside the box. However, the controller based on the KFSMO had a smooth response without any sudden jump, and it was limited to [-50 50] V. Next, the residual of the estimation of the system output is shown in Fig. 8 for both of the controllers based on KSMO and KFSMO. 


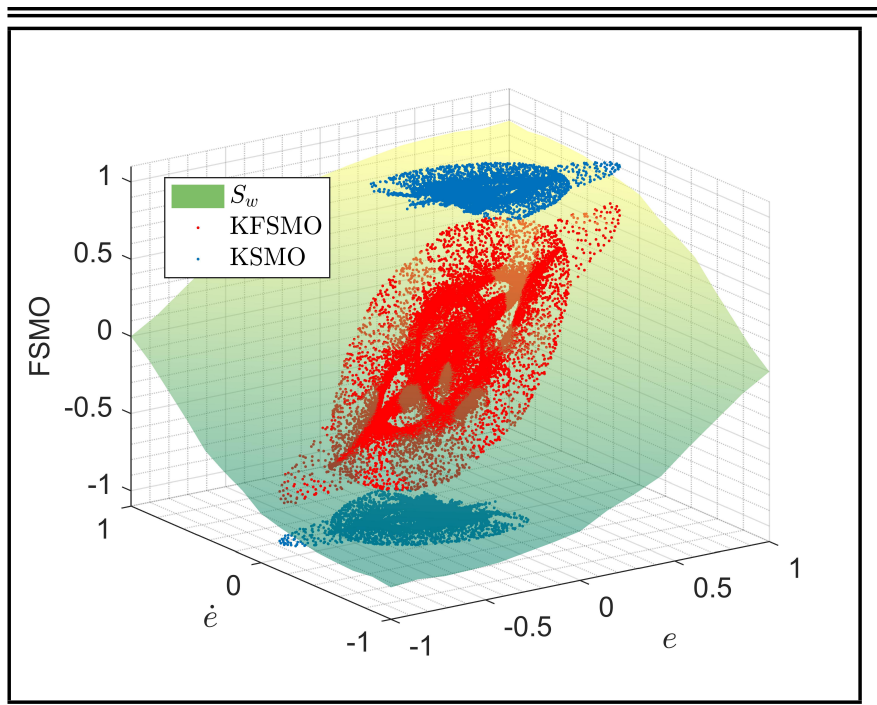

Figure 10. The nonlinear mapping of the fuzzy component.

The comparison of the switching function $\left(S_{\mathrm{w}}(t)\right)$ in Eq. (12) and $\operatorname{sgn}(y-\hat{y})$ in dynamic equation of KSMO of Eq. (4) in the forced vibration is presented in Fig. 9. Figure 9 indicates a stable switching function from the initial time for closed-loop system based on the proposed observer-based system. The chattering behaviour of the control effort for KSMObased controller was due to the sudden jumps that appear in the switching function. However, the smooth nonlinear mapping of the intelligent fuzzy system (see Fig. 4) mimicked the behaviour of the SMO in a jump-less manner. This can be observed in Fig. 10, which presents the mapping of the input linguistic variables to the output variable by means of the rule Table 1.

\section{CONCLUSIONS}

In this note, a Kalman filter is mixed with a sliding mode observer in order to specifically address the unmodelled dynamics of nonlinear nature in the plant and the modelling uncertainties of the state, input, and output matrices. In order to solve the chattering issue of the KSMO and removing the constraint on the time-dependent term in the Lyapunov function, a fuzzy system is assigned to the observer system to imitate the behaviour of the switching function by employing the knowledge of the expert. Then, the proposed state estimation scheme is used in the ADRC problem based on the bounded real Lemma. Given the ADRC, the uncertain terms of the modelling in the system matrices are taken into account and as a result, the stability of the closed-loop system, as well as the conditions for the quadratic convergence, are derived. The solution of the problem is transformed in terms of a convex optimization problem subjected to some LMI/LME constraints.

\section{APPENDIX A}

Lemma A.1. ${ }^{29}$ For real matrices $\Sigma, \Psi, X$ and symmetric matrix $M$, the following first statement can be guaranteed if, and only if, the second one holds for a positive scalar $\zeta$ and $\psi^{T} \psi \leq 1$,

$$
\begin{array}{r}
M+\Sigma \Psi X+X^{T} \Psi^{T} \Sigma^{T}<0 \\
M+\zeta^{-1} \Sigma \Sigma^{T}+\zeta^{-1}\left(\zeta X^{T}\right)(\zeta X)<0 .
\end{array}
$$

Lemma A.2. ${ }^{34}$ For two arbitrary vectors with appropriate dimension such as $p, q$ the following inequality is valid for $\epsilon>0$

$$
p^{T} q+q^{T} p \leq \epsilon p^{T} p+\epsilon^{-1} q^{T} q .
$$

\section{REFERENCES}

1 Du, H., Lam, J., and Sze, K. Y. Non-fragile $\mathrm{H}_{\infty}$ vibration control for uncertain structural systems, Journal of Sound and Vibration, 273 (4), 1031-1045, (2004). https://dx.doi.org/10.1016/S0022-460X(03)00520-0

2 Oveisi, A., and Nestorovic, T. Robust nonfragile observer-based $\mathrm{H}_{2} / \mathrm{H}_{\infty}$ controller, Journal of Vibration and Control, 24 (4), 722-738, (2016). https://dx.doi.org/10.1177/1077546316651548

3 Oveisi, A., and Shakeri, R. Robust reliable control in vibration suppression of sandwich circular plates, Engineering Structures, 116, 1-11, (2016). https://dx.doi.org/10.1016/j.engstruct.2016.02.040

4 Choi, H. H. LMI-Based sliding surface design for integral sliding mode control of mismatched uncertain systems, IEEE Transactions on Automatic Control, 52 (4), 736-742, (2007). https://dx.doi.org/10.1109/TAC.2007.894543

5 Oveisi, A., and Nestorović, T. Robust observer-based adaptive fuzzy sliding mode controller, Mechanical Systems and Signal Processing, 76-77, 58-71, (2016). https://dx.doi.org/10.1016/j.ymssp.2016.01.015

6 Xie, L., and Soh, Y. C. Robust Kalman filtering for uncertain systems, Systems \& Control Letters, 22 (2), 123-129, (1994). https://dx.doi.org/10.1016/0167-6911(94)90106-6

7 Wang, Z., and Unbehauen, H. Robust $\mathrm{H}_{2} / \mathrm{H}_{\infty}$-state estimation for systems with error variance constraints: the continuous-time case, IEEE Transactions on Automatic Control, 44 (6), 1061-1065, (1999). https://dx.doi.org/10.1109/9.763229

8 Utkin, V., and Shi, J. Integral sliding mode in systems operating under uncertainty conditions, Proceedings of 35th IEEE Conference on Decision and Control, Kobe, Japan, (1996). https://dx.doi.org/10.1109/CDC.1996.577594

9 Edwards, C., and Spurgeon, S. K. On the development of discontinuous observers, International Journal of Control, 59 (5), 1211-1229, (1994). https://dx.doi.org/10.1080/00207179408923128

10 Utkin, V. I. Sliding Modes in Control and Optimization, Springer Berlin Heidelberg, Berlin, Heidelberg, (1992).

11 Young, K. D., Utkin, V. I., and Ozguner, U. A control engineer's guide to sliding mode control, IEEE Transactions on Control Systems Technology, 7 (3), 328-342, (1999). https://dx.doi.org/10.1109/VSS.1996.578521

12 Slotine, J.-J. E., and Li, W. Applied nonlinear Control, Applied Spectroscopy, 62 (7), 174-174, (1991). 
13 Keighobadi, J., and Menhaj, M. B. From nonlinear to fuzzy approaches in trajectory tracking control of wheeled mobile robots, Asian Journal of Control, 14 (4), 960-973, (2012). https://dx.doi.org/10.1002/asjc.480

14 Rajamani, R. Observers for Lipschitz nonlinear systems, IEEE Transactions on Automatic Control, 43 (3), 397-401, (1998). https://dx.doi.org/10.1109/9.661604

15 Gao, Z. Active disturbance rejection control: a paradigm shift in feedback control system design, Proceedings of 2006 American Control Conference, Minneapolis, Minnesota, (2006). https://dx.doi.org/10.1109/ACC.2006.1656579

16 She, J.-H., Fang, M., Ohyama, Y., Hashimoto, H., and Wu, M. Improving disturbance-rejection performance based on an equivalent-input-disturbance approach, IEEE Transactions on Industrial Electronics, 55 (1), 380-389, (2008). https://dx.doi.org/10.1109/TIE.2007.905976

17 Wai, R.-J., Lin, C.-M., and Hsu, C.-F. Adaptive fuzzy sliding-mode control for electrical servo drive, Fuzzy Sets and Systems, 143 (2), 295-310, (2004). https://dx.doi.org/10.1016/S0165-0114(03)00199-4

18 Gholami, A., and Markazi, A. H. D. A new adaptive fuzzy sliding mode observer for a class of MIMO nonlinear systems, Nonlinear Dynamics, 70 (3), 2095-2105, (2012). https://dx.doi.org/10.1007/s11071-012-0602-0

19 Chen, K., Paurobally, R., Pan, J., and Qiu, X. Improving active control of fan noise with automatic spectral reshaping for reference signal, Applied Acoustics, 87, 142-152, (2015). https://dx.doi.org/10.1016/j.apacoust.2014.07.003

20 Hasheminejad, S. M., and Oveisi, A. Active vibration control of an arbitrary thick smart cylindrical panel with optimally placed piezoelectric sensor/actuator pairs, International Journal of Mechanics and Materials, 12 (1), 1-16, (2016). https://dx.doi.org/10.1007/s10999-015-9293-2

21 Soize, C. Random matrix theory for modeling uncertainties in computational mechanics, Computer Methods in Applied Mechanics and Engineering, 194 (12-16), 1333-1366, (2005). https://dx.doi.org/10.1016/j.cma.2004.06.038

22 Oveisi, A., Nestorović, T., and Nguyen, N. L. Semianalytical modeling and vibration control of a geometrically nonlinear plate, International Journal of Structural Stability and Dynamics, 17 (4), (2016). https://dx.doi.org/10.1142/S0219455417710031

23 Noël, J. P., and Kerschen, G. Nonlinear system identification in structural dynamics: 10 more years of progress, $\mathrm{Me}$ chanical Systems and Signal Processing, 83, 2-35, (2017). https://dx.doi.org/10.1016/j.ymssp.2016.07.020

24 Mignolet, M. P. and Soize, C. Nonparametric stochastic modeling of linear systems with prescribed variance of several natural frequencies, Probabilistic Engineering Mechanics, 23 (2-3), 267-278, (2008). https://dx.doi.org/10.1016/j.probengmech.2007.12.027
25 Bossi, L., Rottenbacher, C., Mimmi, G., and Magni, L. Multivariable predictive control for vibrating structures: An application, Control Engineering Practice, 19 (10), 1087-1098, (2011). https://dx.doi.org/10.1016/j.conengprac.2011.05.003

26 Adhikari, S., Friswell, M. I., Lonkar, K., and Sarkar, A. Experimental case studies for uncertainty quantification in structural dynamics, Probabilistic Engineering Mechanics, 24 (4), 473-492, (2009). https://dx.doi.org/10.1016/j.probengmech.2009.01.005

27 Gerla, G. Fuzzy logic programming and fuzzy control, Studia Logica, 79 (2), 231-254, (2005). https://dx.doi.org/10.1007/s11225-005-2977-0

28 Yau, H.-T., and Chen, C.-L. Chattering-free fuzzy slidingmode control strategy for uncertain chaotic systems, Chaos, Solitons \& Fractals, 30 (3), 709-718, (2006). https://dx.doi.org/10.1016/j.chaos.2006.03.077

29 Boyd, S., El Ghaoui, L., Feron, E., and Balakrishnan, V. Linear matrix inequalities in system and control theory, Society of Industrial and Applied Mathematics, Philadelphia, (1994).

30 Xie, W. An equivalent LMI representation of bounded real lemma for continuous-time systems, Journal of Inequalities and Applications, 2008 (672905), (2008). https://dx.doi.org/10.1155/2008/672905

31 Lien, C.-H., Cheng, W.-C., Tsai, C.-H., and Yu, K.-W. Nonfragile observer-based controls of linear system via LMI approach, Chaos, Solitons \& Fractals, 32 (4), 1530-1537, (2007). https://dx.doi.org/10.1016/j.chaos.2005.11.092

32 Oveisi, A., and Nestorović, T. Transient response of an active nonlinear sandwich piezolaminated plate, Communications in Nonlinear Science and Numerical Simulation, 45, 158-175, (2017). https://dx.doi.org/10.1016/j.cnsns.2016.09.012

33 Sachdeva, S. K., Nair, P. B., and Keane, A. J. Hybridization of stochastic reduced basis methods with polynomial chaos expansions, Probabilistic Engineering Mechanics, 21 (2), 182-192, (2006). https://dx.doi.org/10.1016/j.probengmech.2005.09.003

34 Pourgholi, M., and Majd, V. J. A new non-fragile $\mathrm{H}_{\infty}$ proportional-integral filtered-error adaptive observer for a class of non-linear systems and its application to synchronous generators, The Proceedings of the Institution of Mechanical Engineers, Part I: Journal of Systems and Control Engineering, 225 (1), 99-112, (2011). https://dx.doi.org/10.1243/09596518JSCE1061 